\title{
Measurement of a branching ratio of bound and continuum-state $\beta^{-}$decay
}

\author{
C. Scheidenberger ${ }^{a}$, F. Bosch ${ }^{a}$, H. Geissel ${ }^{a, b}$, T. Ohtsubo ${ }^{a, c}$, \\ F. Attallah ${ }^{a}$, K. Beckert ${ }^{a}$, P. Beller ${ }^{a}$, D. Boutin ${ }^{a}$, T. Faestermann ${ }^{d}$, \\ B. Franczak ${ }^{a}$, B. Franzke ${ }^{a}$, M. Hausmann ${ }^{a}$, M. Hellström ${ }^{a}$, \\ E. Kaza ${ }^{a}$, P. Kienle ${ }^{d, e}$, O. Klepper ${ }^{a}$, H.-J. Kluge ${ }^{a}$, C. Kozhuharov ${ }^{a}$, \\ Yu. A. Litvinov ${ }^{a}$, L. Maier ${ }^{d}$, M. Matos ${ }^{a}$, G. Münzenberg ${ }^{a, f}$, \\ F. Nolden ${ }^{a}$, Yu. N. Novikov ${ }^{a, g}$, M. Portillo ${ }^{a}$, T. Radon ${ }^{a}$, \\ J. Stadlmann ${ }^{a, b}$, M. Steck ${ }^{a}$, T. Stöhlker ${ }^{a, h}$, K. Sümmerer ${ }^{a}$, \\ K. Takahashi ${ }^{a}$, H. Weick ${ }^{a}$, M. Winkler ${ }^{a, b}$, and T. Yamaguchi ${ }^{a, i}$ \\ ${ }^{a}$ GSI, 64291 Darmstadt, Germany \\ ${ }^{b}$ Justus-Liebig-Universität, 35392 Gießen, Germany \\ ${ }^{c}$ Niigata University, Niigata 950-2181, Japan \\ $d$ Technische Universität München, 85748 Garching, Germany \\ ${ }^{e}$ Stefan Meyer Institut der OAW, 1090 Wien, Austria \\ $f$ Johannes Gutenberg-Universität Mainz, 55099 Mainz, Germany \\ $g$ St. Petersburg Nuclear Physics Institute, Gatchina 188300, Russia \\ ${ }^{h}$ Johann-Wolfgang-Goethe Universität, 60486 Frankfurt, Germany \\ ${ }^{i}$ Saitama University, Saitama 338-8570, Japan
}

The first measurement of a ratio $\lambda_{\beta_{b}} / \lambda_{\beta_{c}}$ of bound-state $\left(\lambda_{\beta_{b}}\right)$ and continuumstate $\left(\lambda_{\beta_{c}}\right) \beta^{-}$-decay rates for the case of bare ${ }^{207} \mathrm{Tl}^{81+}$ ions is reported. Since $\beta^{-}$-decay to a bound final state of the released electron is the time-reversed process of electron capture, the measurement is an approach to test $\beta$-decay theory. The experimental result, $\lambda_{\beta_{b}} / \lambda_{\beta_{c}}=0.188(18)$, is in excellent agreement with the theoretical value of $0.171(1)$. 


\section{Introduction}

Weak interactions, mediated by charged and neutral currents, are described by the electroweak theory. This theory was developed along the lines of the pioneering work of Glashow, Weinberg, and Salam in the 1960s. Before the discovery of the neutral $Z^{0}$ gauge boson in the 1980s, all phenomena observed in weak decays could be explained within the theoretical framework based on Fermi's theory of $\beta$-decay. Still today, it can be understood as the low-energy approximation of the electro-weak theory. Vast theoretical and experimental $\beta$-decay studies have been carried out, but still many open questions remain, and one of them is addressed in the present study, where the problem of time-reversal is addressed. For this purpose, $\beta^{-}$-decay into bound final states of the created electron has been studied, which is the time-mirrored process of orbital electron capture (EC). While the branching ratio of EC and continuum $\beta^{+}$-decay, $R\left(\beta^{+}\right)=\lambda_{E C} / \lambda_{\beta^{+}}$, has been explored extensively by theory [1] and a multitude of measurements [2], the corresponding ratios of the timemirrored $\beta^{-}$-decay (see fig. 1) $\mathrm{R}\left(\beta^{-}\right)=\lambda_{\beta_{b}} / \lambda_{\beta_{c}}$, for electron emission to bound $\left(\beta_{b}\right)$ and continuum states $\left(\beta_{c}\right)$, respectively, have not yet been addressed by experiments. Besides

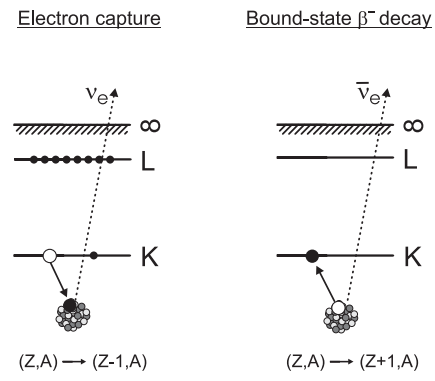

Figure 1: Schematic view of the decay of a nucleus with atomic number $Z$ and mass number $A$ by capture of a K-shell electron (left) and the time-reversed process of $\beta^{-}$-decay, where the released electron is captured into a bound final state in the K-shell (right).

this fundamental interest, the study is also important for astrophysical applications [3], where beta-decay strengths define the time scale of nuclear transmutations, determine the pathways of stellar nucleosynthesis and also the final abundance of atomic nuclei in our solar system and in the stars.

In this contribution, the performed experiments are briefly described, first results on the branching ratio $R\left(\beta^{-}\right)$of bare ${ }^{207} \mathrm{Tl}^{81+}$ are presented and compared to theoretical predictions, and new results on the production and half-life of the bare isomeric state ${ }^{207 m} \mathrm{Tl}^{81+}$ are presented. 


\section{Experiment}

Two experiments have been carried out under almost equal conditions employing relativistic secondary beams at the SIS-FRS-ESR facility of GSI. An essential prerequisite for the desired measurements is the production, monoisotopic separation and storage of bare radioactive heavy ions and the simultaneous observation of the mother ions and their $\beta_{b}$ daughters as a function of time. This is achieved with time-resolved Schottky Mass Spectrometry (SMS) [4]. To maximize the relative yield of $\beta_{b}$ decays, the $Q$-value of $\beta^{-}$-decay should be as small as possible. On the other hand, the $Q$-value has to be sufficiently large in order to avoid too long half-lives and to resolve the signals of mother- and $\beta_{b}$-daughter in the Schottky frequency spectrum. As a compromise, these somewhat conflicting constraints led to the choice of bare ${ }^{207} \mathrm{Tl}^{81+}$ as mother ions with a neutral-atom $\beta^{-}$half-life of $4.77 \mathrm{~min}$ and a neutral-atom $Q$-value of $1.418 \mathrm{MeV}[5]$. The $1 / 2^{+} \mathrm{g}$. s. of neutral ${ }^{207} \mathrm{Tl}^{0}$ decays by a non-unique first-forbidden transition with almost $100 \%$ to the $1 / 2^{-} \mathrm{g}$. s. of ${ }^{207} \mathrm{~Pb}$.

The ${ }^{207} \mathrm{Tl}$ was produced by fragmentation reactions of an $838 A \mathrm{MeV}{ }^{208} \mathrm{~Pb}$ beam in a $4 \mathrm{~g} / \mathrm{cm}^{2}$ thick beryllium production target at the entrance of the fragment separator FRS [6]. The reaction cross section is about 30 mbarn [7]. The cocktail of the fragments was analyzed by the FRS using a two-stage magnetic separation with a wedge shaped degrader in between $(B \rho-\Delta E-B \rho$-method [6]). In this way isotopic separation of ${ }^{207} \mathrm{Tl}^{81+}$ at the exit slit of the FRS was achieved. Special care was taken to separate bare ${ }^{207} \mathrm{Tl}^{81+}$ ions from nearby $\mathrm{H}$-like ${ }^{207} \mathrm{~Pb}^{81+}$ ions (the $\beta_{b}$ daughters of ${ }^{207} \mathrm{Tl}^{81+}$ ) by using a sufficiently thick degrader (35\% of the range). Thereby the fraction of ${ }^{207} \mathrm{~Pb}^{81+}$ ions injected at an energy of $400.5 \mathrm{~A} \mathrm{MeV}$ into the ESR [8] was reduced to only a few percent of the corresponding bare ${ }^{207} \mathrm{Tl}^{81+}$ ions. After injection of an almost pure bunch of bare ${ }^{207} \mathrm{Tl}^{81+}$ ions into the ESR, electron cooling [9] was applied, which generates brillant beams of highest phase space density, i. e. of small transverse emittance and velocity spread $\left(\delta v / v=10^{-5} \ldots 10^{-7}\right)$. With an electron current of $200 \mathrm{~mA}$ it enabled $1 / e$-storage times of about 40 minutes for these heavy highly-charged ions at a residual gas pressure of about $10^{-11}$ mbar. However, for 'hot' fragments with a large initial velocity spread, as produced by fragmentation, electron cooling requires a rather long time in the order of one to two minutes (in this experiment stochastic pre-cooling was not yet available). The reason is that the cooling-force decreases as $\Delta v^{-3}$, where $\Delta v$ denotes the difference of the ion velocity and the velocity of the cooler electrons. After complete electron cooling, all stored ions circulate at almost the same velocity due to the repeated interaction with the cold cooler electrons, which they traverse with a circulation frequency of about $2 \mathrm{MHz}$. The coasting ions induce a Schottky-noise signal when passing the pair of capacitive pickup plates mounted inside the storage-ring aperture. When Fourier-transformed, each stored ion species produces lines ('Schottky lines') at distinct frequencies corresponding to the revolution frequencies of the ion species (sc. harmonics), determined by their mass-to-charge ratio $m / q$.

The data were recorded and processed using a spectrum analyzer. Starting at the time of injection of an ion bunch, the signals of the Schottky-pickups were recorded for subsequent time intervals of $320 \mathrm{~ms}$ (or $640 \mathrm{~ms}$ ) duration, on-line analyzed by a FastFourier-Transform (FFT), and the resulting frequency spectra were stored. From these 
signals, taken at the $30^{\text {th }}$ harmonic of the revolution frequency $f=1.978 \mathrm{MHz}$ of the ions, the frequency of an internal local oscillator operating at 59,340,500 $\mathrm{Hz}$ was subtracted. The resulting spectra were stored covering a $1 \mathrm{kHz}$-band and comprising 640 channels, thus yielding a resolution of $1.56 \mathrm{~Hz}(3.12 \mathrm{~Hz})$ per channel. In order to increase the signal-to-noise ratio, the data were averaged over 100 single spectra, corresponding to $32 \mathrm{~s}(64 \mathrm{~s})$, after a minor correction for slow frequency shifts caused by small drifts of the power supplies of the ESR. In total, 4000 single spectra of $320 \mathrm{~ms}$ (640 ms) duration were accumulated, corresponding to an overall measuring time of $1280 \mathrm{~s}(2560 \mathrm{~s})$.

The areas of the Schottky lines (corresponding to the integrated noise power) yield at each time interval the number $N$ of the corresponding ion species, as these intensities are proportional to $q^{2} \cdot N$. As can be seen from fig. 2, a bunch containing about $1900{ }^{207} \mathrm{Tl}^{81+}$ ions was injected, containing a few (about 20) ${ }^{207} \mathrm{~Pb}^{81+}$ ions. The time-resolved Schottky spectroscopy was applied solely to the small frequency range comprising ${ }^{207} \mathrm{Tl}^{81+}$ mother ions and the near-lying ${ }^{207} \mathrm{~Pb}^{81+}$ bound-state $\beta^{-}$-decay daughter ions. The time evolution of the number $N_{\mathrm{Tl}}(t)$ of the bare ${ }^{207} \mathrm{Tl}^{81+}$ mother ions and of the number $N_{\mathrm{Pb}}(t)$ of the $\mathrm{H}$-like ${ }^{207} \mathrm{~Pb}^{81+} \beta_{b}$ daughter ions is given by:

$$
\begin{aligned}
d N_{\mathrm{Tl}}(t) / d t & =-\left[\lambda_{\beta_{c}}+\lambda_{\beta_{b}}+\lambda_{\text {loss }}(\mathrm{Tl})\right] \cdot N_{\mathrm{Tl}}(t) \\
d N_{\mathrm{Pb}}(t) / d t & =\lambda_{\beta_{b}} N_{\mathrm{Tl}}(t)-\lambda_{\text {loss }}(\mathrm{Pb}) \cdot N_{\mathrm{Pb}}(t)
\end{aligned}
$$

where $\lambda_{\beta_{c}}$ and $\lambda_{\beta_{b}}$ are the decay probabilities to continuum and bound states, respectively, and where $\lambda_{\text {loss }}$ denotes an additional reduction for both, bare Tl- and H-like $\mathrm{Pb}$-ions, due to unavoidable beam losses in the machine and (atomic) charge-changing processes in the electron cooler or the residual gas. In previous experiments $[10,11]$ it has been shown that for neighbouring bare and H-like heavy ions the corresponding $\lambda_{\text {loss }}$ constants are nearly equal: compared with bare ions, the slightly smaller electron capture rate in the cooler for H-like ions is, accidentally, nearly compensated by an additional ionization rate in the residual gas for a pressure of about $10^{-11} \mathrm{mbar}$ and for a gas containing mainly hydrogen. It seemed reasonable, therefore, to assume the same loss constant, $\lambda_{\text {loss }}(\mathrm{Tl})=\lambda_{\text {loss }}(\mathrm{Pb})=\lambda_{\text {loss }}$ for both ion species. Introducing a total decay constant $\lambda=\lambda_{\beta_{c}}+\lambda_{\beta_{b}}+\lambda_{\text {loss }}$, the solutions of eqs. 1 and 2 are:

$$
\begin{aligned}
N_{\mathrm{Tl}}(t)= & N_{\mathrm{Tl}}(0) \cdot \exp (-\lambda t) \\
N_{\mathrm{Pb}}(t)= & N_{\mathrm{Tl}}(0) \cdot \frac{\lambda_{\beta_{b}}}{\lambda_{\beta_{c}}+\lambda_{\beta_{b}}} \cdot\left[\exp \left(-\lambda_{\text {loss }} t\right)-\exp (-\lambda t)\right] \\
& +N_{\mathrm{Pb}}(0) \cdot \exp \left(-\lambda_{\text {loss }} t\right)
\end{aligned}
$$

where $N_{\mathrm{Tl}}(0), N_{\mathrm{Pb}}(0)$ are the corresponding ion numbers present at the time of injection $(t=0)$. Figure 2 shows as a function of time the number of ${ }^{207} \mathrm{Tl}^{81+}$ mother ions and their $\beta_{b}$ daughters, H-like ${ }^{207} \mathrm{~Pb}^{81+}$ ions, taken over $1280 \mathrm{~s}$. In long-time runs the number of $\mathrm{Tl}$ ions could be finally reduced to one single ion. Therewith the Schottky-noise intensity corresponding to one, two, three, ... Tl ion(s) was determined. Since the charge state $q=81$ is identical for both ion species, the same calibration could be applied for the $\mathrm{Pb}$ ions.

The data analysis proceeded via the following steps: First, long-time runs exceeding more than $40 \mathrm{~min}$. were exploited to determine $\lambda_{\text {loss }}$ by a separate fit to the $\mathrm{Pb}$ data from 
the time regime after the complete decay of the $\mathrm{Tl}$ ions. Then, from a fit of all available $\mathrm{Tl}$ data according to eq. 3 , the initial number of $\mathrm{Tl}$ ions, $N_{\mathrm{Tl}}(0)$, the total decay constant $\lambda$ in the laboratory system and, hence, $\lambda_{\beta_{c}}+\lambda_{\beta_{b}}=\lambda-\lambda_{\text {loss }}$ were determined. Finally, the remaining parameters $\lambda_{\beta_{b}}$ and $N_{\mathrm{Pb}}(0)$ were obtained from a fit to the $\mathrm{Pb}$ data according to eq. 4 . The first three points at $16 \mathrm{~s}, 48 \mathrm{~s}$ and $80 \mathrm{~s}$ show significant deviations from the exponential decay curve, caused by the long time needed to cool the hot $\mathrm{Tl}$ fragments employing electron cooling. Therefore these points were excluded from the fits.

\section{Results and discussion}

The results for the decay constants in the laboratory system, taken from the fits to the Schottky spectra of the ${ }^{207} \mathrm{Tl}^{81+}$ mother ions and their $\beta_{b}$ daughter ions, ${ }^{207} \mathrm{~Pb}^{81+}$, are: $\lambda=22.8(4) \cdot 10^{-4} \mathrm{~s}^{-1}, \lambda_{\text {loss }}=3.8(7) \cdot 10^{-4} \mathrm{~s}^{-1}, \lambda_{\beta_{b}}=3.0(2) \cdot 10^{-4} \mathrm{~s}^{-1}$, and, therewith, $\lambda_{\beta_{c}}=\lambda-\lambda_{\text {loss }}-\lambda_{\beta_{b}}=16.0(8) \cdot 10^{-4} \mathrm{~s}^{-1}$. After transformation into the rest frame (r.f.) of the ions according to $\lambda$ (r.f.) $=\gamma \cdot \lambda$, we obtain with a Lorentz factor $\gamma=1.430(1)$ (determined from the voltage of the electron cooler) the following decay probabilities:

$$
\begin{aligned}
\lambda(\exp ) & =32.6(6) \cdot 10^{-4} \mathrm{~s}^{-1} \\
\lambda_{\beta b}(\exp ) & =4.29(29) \cdot 10^{-4} \mathrm{~s}^{-1} \\
\lambda_{\beta c}(\exp ) & =22.9(12) \cdot 10^{-4} \mathrm{~s}^{-1} \\
{\left[\lambda_{\beta_{b}} / \lambda_{\beta_{c}}(\exp )\right.} & =0.188(18)
\end{aligned}
$$

The obtained branching ratio is depicted in the right part of fig. 2 .

The detection technique employed here does not allow to resolve transitions to individual atomic shells of the bound electron states, however the properties of the atomic structure are of minor importance, because the capture into bound states is dominated by the overlap of the atomic wave functions with the nucleus. Therefore, the above quoted $\lambda_{\beta_{b}}$ value comprises the sum of all transition probabilities to bound states; we calculate that about $80 \%$ of the $\beta_{b}$ transitions pass directly into the $1 \mathrm{~s}_{1 / 2}$ state of ${ }^{207} \mathrm{~Pb}$. This can be easily estimated from the following argument: with non-relativistic hydrogen-like wave function the probability to find an electron at the nucleus $(R=0)$ scales approximately like $(Z \alpha / n)^{3}$, where $Z$ is the atomic number, $\alpha \simeq 1 / 137$, and $n$ is the principal quantum number. Already from this simple scaling law one finds that approximately $80 \%$ are captured to the K-shell.

It is now interesting to compare the decay properties of the bare nucleus with those of the neutral atom. First of all and most important, the newly available phase space in bare nuclei opens up the possibility for the new decay channel of bound-state $\beta^{-}$decay. The electron states in the empty atomic shell -Pauli-blocked in the atom- are now available as final states. Thus a high degree of ionization is a prerequisite for a substantial branching ratio of this decay mode. The present data have important applications in nuclear astrophysics, because the pathways of hot stellar nucleosynthesis might be altered drastically by a high ionic charge state $q$, because it enables new branchings due to enhanced bound-state $\beta^{-}$decays. Vice versa: from measured nuclear abundances in the vicinity of those branching points the mean ionic charge state $\langle q\rangle$ and, thus, the stellar 

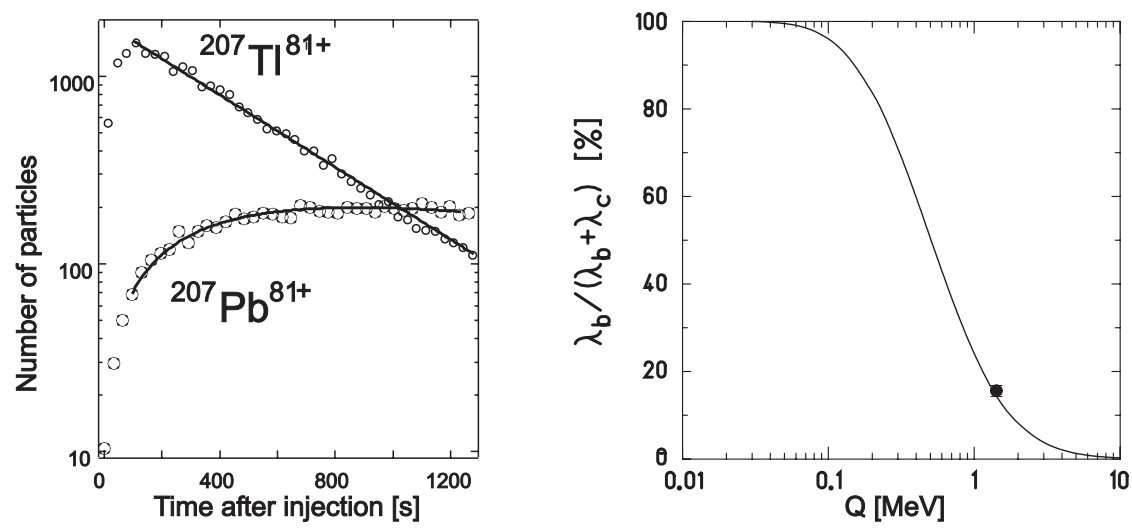

Figure 2: Left: Intensities of bare mother $\left({ }^{207} \mathrm{Tl}^{81+}\right)$ and H-like bound- $\beta$-decay daughter ions $\left({ }^{207} \mathrm{~Pb}^{81+}\right)$ as a function of storage time displayed together with the fit functions as described in the text. Right: percentage fraction of the bound- $\beta$ decay rate of bare ions with atomic number $Z=81$. Solid line: calculation according to ref. [12] as a function of $Q$-value. Data point: measured value of the present experiment for ${ }^{207} \mathrm{Tl}^{81+}$.

temperature may be constrained, e. g. for the s-process [13], provided $R\left(\beta^{-}\right)$is known as a function of $q$.

As can be seen from the right part of fig. 2, the absolute magnitude of the Q-value determines sensitively the ratio of bound and continuum decay (as is the case for the time-mirrored processes electron capture and $\beta^{+}$-decay): the branching ratio is maximized for small Q-values. In some cases, as for instance in the case of bare ${ }^{163} \mathrm{Dy}$ [10], where the $Q$-value is smaller than the K-shell binding energy of the electron in the daughter nucleus, bound-state $\beta$-decay is the only energetically possible and thus the exclusively possible decay mode.

In addition to that, there are two effects in bare nuclei, which need consideration: the missing electrons lead to a reduced screening of the nuclear charge and the $Q$-value is altered as compared to the neutral atom. The first effect, the absence of electrons at the nuclear surface leads to a "higher" charge and thus to a slightly enhanced $\beta^{-}$-decay probability, which can be taken from tabulated values and which is calculated from the integrated Fermi function $f$ with and without screening [1]. In the present case the total decay probability is increased by $2.5 \%$. The other effect is the modified Q-value as compared to neutral atoms. In neutral ${ }^{207} \mathrm{Tl}$, the Q-value for continuum $\beta^{-}$-decay is $Q_{\beta_{c}}^{q=0}=1,417.9 \mathrm{keV}$. As can be found in equations (2)..(7) in ref. [14] the $Q$-value is modified for the bare nucleus by the difference of the total binding energies of all missing electrons of mother $(Z=81)$ and daughter nucleus $(Z=82)$, which amounts to $\Delta B_{e, \text { tot }}(82,81)=17.4 \mathrm{keV}$, thus leading to $Q_{\beta_{c}}^{q=Z}=1,400.5 \mathrm{keV}$. Calculating $f$ for this modified value leads to a reduction of the decay rate by $4.6 \%$. Both effects together lead finally to a reduction by $2.2 \%$ and the calculated decay rate for continuum $\beta^{-}$-decay 
is $23.7 \pm 0.2 \cdot 10^{-4} \mathrm{~s}^{-1}$, which is in agreement with the experimentally observed value of $22.9 \pm 1.2 \cdot 10^{-4} \mathrm{~s}^{-1}$. These values have to be compared with the decay rate of neutral ${ }^{207} \mathrm{Tl}^{0}, \lambda\left({ }^{207} \mathrm{Tl}^{0}\right)=24.2 \cdot 10^{-4} \mathrm{~s}^{-1}[5]$, which is almost solely due to continuum $\beta^{-}$decay. Whereas the continuum decay rate is slightly reduced $(-2.2 \%)$, the total decay rate is enhanced by the bound-state $\beta$-decay, which accounts for $17 \%$. Thus, the new decay channel has a much larger influence than the modification of the $Q$-value.

The branching ratio $R\left(\beta^{-}\right)$renders -besides small corrections- the ratio of all bound electron states of the daughter atoms and of the densities for continuum electron states at the origin, as comprised in the Fermi function. We calculate, by using 'allowed spectra' of the continuum electrons and antineutrinos for the Fermi function in the continuum $\beta^{-}$-decay, and relativistic hydrogen-like wave functions of the bound states (up to and including $6 \mathrm{~s}_{1 / 2}$ ) for bound-state $\beta^{-}$-decay to $\mathrm{H}$-like ${ }^{207} \mathrm{~Pb}^{81+}$ (see also [12]):

$$
\begin{aligned}
\lambda_{\beta_{b}}(\text { theo }) & =4.06(2) \cdot 10^{-4} \mathrm{~s}^{-1} \\
\lambda_{\beta_{c}}(\text { theo }) & =23.7(2) \cdot 10^{-4} \mathrm{~s}^{-1} \\
{\left[\lambda_{\beta_{b}} / \lambda_{\beta_{c}}\right](\text { theo }) } & =0.171(1)
\end{aligned}
$$

in excellent agreement with the experimental values quoted in eq. (5). It must be noted here, however, that the transition of our current interest is non-unique first-forbidden. In principle, therefore, the above statement based on the 'normal approximation' [15], i. e., the assumption of the 'allowed' spectra, is not always valid. In some cases, non-negligible energy dependencies that are different for different matrix elements may appear. In the present case of ${ }^{207} \mathrm{Tl}$, the deviation from the allowed spectra seems to be small, however (some percent at maximum $[16,17]$ ), thus justifying the approximation made.

In another experiment, which was carried out under very similar conditions, stochastic pre-cooling was used, thus leading to overall cooling times of about six seconds only. Therefore the properties of the short-lived isomeric state in ${ }^{207} \mathrm{Tl}^{81+}$ (half-life of the neutral atom $T_{1 / 2}=1.33 \pm 0.11 \mathrm{~s}[18,19]$ ) could be investigated. From the decay curves of ground and isomeric state, as shown in an example of one run in fig. 3, the halflife and the isomeric ratio can be extracted. The measured half-live of the bare isomer $T_{1 / 2 \text {,exp. }}\left({ }^{207 m} \mathrm{Tl}^{81+}\right)=1.47 \pm 0.32 \mathrm{~s}$ is slightly larger than that of the neutral atom. The origin is the missing electron cloud and thus the impossibility of deexcitation by the emission of conversion electrons. Taking this into account, one obtains a calculated half-live $\left.T_{1 / 2 \text {,calc. }}{ }^{207 m} \mathrm{Tl}^{81+}\right)=1.52 \pm 0.13 \mathrm{~s}$, which is in agreement with the experimental value. The isomeric ratio of a certain nuclide $R=\frac{I_{m}(0)}{I_{g}(0)+I_{m}(0)}$ is the fraction of isomeric states produced in the fragmentation reaction $\left(I_{m}(0)\right)$ relative to the number of all fragments (ground state $I_{g}(0)$ and isomeric state) of this nuclide. It is obtained by extrapolation of the the decay curves of ground and isomeric state from the measured data points to the moment of production $t=0$, as indicated in fig. 3 by the dashed lines. For the present case, $R\left({ }^{207 m} \mathrm{Tl}\right)=0.15 \pm 0.04$. This fraction is comparable to isomeric ratios measured in other experiments $[20,4]$ and is compared to model calculations along the lines of ref. [21] in the right part of fig. 3. As can be seen, the agreement with experimental data is reasonable. 

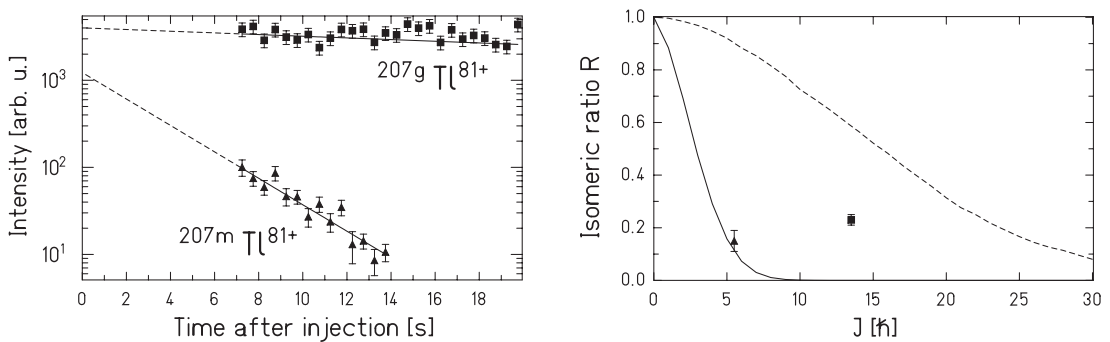

Figure 3: Left: Intensities of the ground and isomeric state of bare ${ }^{207} \mathrm{Tl}$ nuclei as a function of storage time. The solid lines show the fit to the data, the dashed lines indicate the extrapolation to the point of production $(\mathrm{t}=0)$. Right: Probability for isomer production expressed in terms of the isomeric ratio (see text) for ${ }^{207} \mathrm{Tl}$ (triangle, solid line) and for ${ }^{149} \mathrm{Dy}$ (square, from ref. [4], dashed line) as a function of the spin of the isomeric states.

\section{Summary}

In summary, for the first time we have directly observed the bound-state $\beta^{-}$decay. It is the merit of radioactive beams, that an isotope with a suitable $Q$-value can be selected such that the observation becomes possible. The studied decay mode is the time-mirrored electron capture, which is one of the fundamental weak-interaction decay modes with charged currents. A branching ratio of bound and continuum $\beta$-decay in ${ }^{207} \mathrm{Tl}$ was measured, which is the analogue of the extensively studied branching ratio of $\beta^{+}$-decay and electron capture. Within an uncertainty of a few percent, the result confirms calculations based on the assumption of the 'allowed' spectra for the investigated non-unique firstforbidden $\beta^{-}$transition of bare ${ }^{207} \mathrm{Tl}^{81+}$. Therefore the experimental result strengthens the reliability of astrophysical s-process calculations, where such branching ratios enter. The use of stochastic cooling allows to investigate also the half-life of short-lived isomeric states as shown in the case of ${ }^{207 m} \mathrm{Tl}$ and to study the fragmentation-reaction mechanism.

\section{Acknowledgement}

We thank the operators and engineers of the GSI accelerators for providing us with an excellent ${ }^{208} \mathrm{~Pb}$ beam over many days. We are very much indebted to K.-H. Behr, A. Brünle, C. Karagiannis, and A. Schwinn for valuable help. This work was supported by BMBF under contract number 06 TM970, by DFG under contract number SFB 375-95, and by WTZ under contract number RUS-01/696. T. O. acknowledges support from the Ministry of Education, Science and Culture of Japan through the overseas fellowship. 


\section{References}

[1] H. Behrens and J. Jänecke in Numerical Tables for Beta-Decay and Electron Capture, Landolt-Börnstein New Series, Vol. 4 (1969).

[2] W. Bambynek et al., Rev. Mod. Phys. 49, 77 (1977).

[3] F. Bosch, contribution to these proceedings.

[4] B. Franzke, Phys. Scripta T59, 176 (1995),

O. Klepper, Nucl. Phys. A626, 199c (1997),

Yu. A. Litvinov et al., Phys. Lett. B573, 80 (2003), and

Yu. A. Litvinov et al., Nucl. Phys. A734, 473 (2004).

[5] M. R. Schmorak, Nucl. Data Sheets 43, 383 (1984).

[6] H. Geissel et al., Nucl. Instr. Meth. B204, 71 (2003).

[7] K. Sümmerer et al., Phys. Rev. C61, 034607 (2000).

[8] B. Franzke, Nucl. Instr. Meth. B24/25, 18 (1987).

[9] M. Steck et al., Nucl. Instr. Meth. A532, 357 (2004).

[10] M. Jung et al., Phys. Rev. Lett. 69, 2164 (1992).

[11] F. Bosch et al., Phys. Rev. Lett. 77, 5190 (1996).

[12] K. Takahashi et al., At. Data Nucl. Data Tabl. 36, 375 (1987) and

K. Takahashi et al., Nucl. Phys. A404, 578 (1983).

[13] F. Kaeppeler et al., Annu. Rev. Nucl. Part. Science 48, 175 (1998).

[14] F. Bosch, Phys. Scripta T59, 221 (1995).

[15] E. J. Konopinski and M. E. Rose in Alpha-, Beta- and Gamma-Ray spectroscopy, Ed. K. Siegbahn, p. 1327, North-Holland (1966).

[16] J. M. Trischuk et al., Nucl. Phys. A90, 33 (1967).

[17] J. Eichler, Z. Phys. 171, 463 (1963).

[18] R. B. Firestone et al., Table of Isotopes, John Wiley, New York (1996).

[19] E. Browne, Nucl. Data Sheets 88, 29 (1999).

[20] M. Pfuetzner et al., Phys. Lett. B444, 32 (1998).

[21] M. de Jong et al., Nucl. Phys. A613, 435 (1997). 
